\title{
Autoestima: Actualización y Mantenimiento. Un Modelo Teórico con Aplicaciones en Terapia
}

\section{Self-Esteem: Update and Maintenance. A Theoretical Model with Applications in Therapy}

\author{
Antonio Duro Martín \\ Práctica Privada, España
}

\begin{abstract}
Resumen: Conceptuando la autoestima como la resultante de la comparación entre dos esquemas de yo: yo modelo y yo percibido (autoconcepto), se propone un modelo de carácter sistémico, integrado y analítico para explicar cómo aquel constructo se actualiza y mantiene en tiempo real. Su estructura teórica incluye elementos de dos tipos: componentes -antecedentes situacionales, representaciones y estados mentales- y procesos cognitivos -deducción, interpretación, atribución. Dentro de su funcionamiento destacan las ocasiones de autoestima -situaciones antecedentes desencadenantes-, el papel que juega el esquema yo modelo como principio para derivar diversos bloques de información, las experiencias finales producidas por la autoestima, y un proceso de retroalimentación negativa para corregir las entradas al sistema. El modelo se fundamenta en resultados de investigación en este campo, y es coherente con otras diversas teorías y constructos psicológicos. Se perfilan sus aplicaciones clínicas en psicopatología y psicoterapia, en especial la posibilidad de desarrollar una terapia propia.
\end{abstract}

Palabras claves: autoestima, baja autoestima, modelo, teoría, terapia, autoconcepto.

\begin{abstract}
By conceptualizing self-esteem as the result of the comparison between two ego schemas: the model-ego and the perceived-ego (self-concept), a systemic, integrated, and analytical model is proposed to explain how that construct is updated and maintained in real time. Its theoretical structure includes two types of elements: components -situational antecedents, representations and mental states-, and cognitive processes -deduction, interpretation and attribution. Within its operation are the self-esteem occasions -situational triggers-, the role played by the model-ego as a principle from which to draw various blocks of information, the final experiences produced by self-esteem, and a process of negative
\end{abstract}

\footnotetext{
Antonio Duro Martín (iD https://orcid.org/0000-0001-5765-8776 es Doctor en Psicología, Psicólogo Especialista en Psicología Clínica, ejerce como psicólogo clínico en su consulta privada Gabinete de Psicología Colmenar.

Para citar este artículo: Duro, A. (2021). Autoestima: Actualización y Mantenimiento. Un Modelo Teórico con Aplicaciones en Terapia. Clínica Contemporánea, 12(3), Artículo e23. https://doi.org/10.5093/cc2021a16

La correspondencia sobre este artículo debe enviarse al autor al email: anduma@cop.es

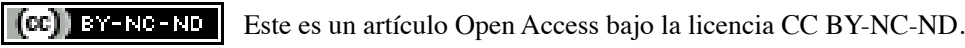


feedback to correct inputs to the system. The model is based on research results in this field and is consistent with various other psychological theories and constructs. Its clinical applications in psychopathology and psychotherapy are outlined, particularly the possibility of developing one's own therapy.

Keywords: self-esteem, low self-esteem, model, theory, therapy, self-concept

La relevancia de la autoestima se pone de manifiesto por la cantidad de publicaciones académicas que ha venido generando. Hay más de doce mil referencias para "self-esteem" y más de trescientas cincuenta para la combinación "self-esteem + low", como términos de búsqueda para el campo "título" en la base datos PsycINFO, a julio de 2020. Cualitativamente, su importancia se revela por la multitud de teorías sobre este constructo (Maxwell y Bachkirova, 2010). Existen, además, recientes metaanálisis que le vinculan con la orientación sexual (Bridge et al., 2019), las relaciones sociales (Harris y Orth, 2020), la identidad de estatus (Ryeng et al., 2013), el tratamiento de pacientes anoréxicos (Kastner et al., 2019) y las diferencias entre hombre y mujer (Zuckerman et al., 2016); y metaanálisis que estudian su desarrollo durante el ciclo vital (Orth et al., 2018) y cambios desde la infancia (Huang, 2010).

Por otro lado, la baja autoestima y psicopatología asociada se extiende por toda la población, afectando a niños (Wanders et al., 2008), adolescentes (Taylor y Montgomery, 2007) y adultos (Steiger et al., 2015). Presenta comorbilidad con cuadros de ansiedad (Pyszczynski et al., 2004) y depresión (Steiger et al., 2015), trastornos alimenticios (Chang 2020; Shiina et al., 2005), personalidad (Jacob et al., 2010), y algunas psicosis (Hall y Tarrier, 2003). De ahí la variedad de terapias desarrolladas desde enfoques clínicos clásicos y de última generación.

Sin embargo, tal abundancia de investigaciones y terapias adolece de una excesiva fragmentación, dando la impresión de que todas ellas aluden a unos mismos factores subyacentes que, sin embargo, quedan implícitos. Haciéndonos cuestión de este problema, el objeto de este trabajo es proponer un modelo integrado de autoestima, de carácter sistémico y con una descripción de procesos muy analítica -a fin de posibilitar su posterior aplicación clínica-, en donde se expliciten los antecedentes, representaciones y estados mentales constituyentes en su actualización y mantenimiento, fundamentando su estructura teórica en resultados empíricos previos.

Antes de exponer el modelo precisaremos su objeto material y estadio de desarrollo. Se trata de un modelo cognitivo-conductual de carácter factual o interpretado para explicar la actualización y mantenimiento de la autoestima; no es un modelo para explicar la autoestima. Se encuentra en una fase preliminar de construcción teórica y, al presente, ofrece una definición general (de carácter más abstracto) de sus componentes y procesos, así como una secuencia de relaciones no arbitrarias entre ellos. Pese a su actual carácter heurístico, el modelo cumple, como se comprobará, con aquellos criterios metodológicos exigibles (Bunge, 2000) en una fase teórica preliminar, a saber: unidad conceptual e interpretabilidad empírica (criterios semánticos); así como consistencia externa, alcance, profundidad y capacidad unificadora (criterios gnoseológicos), haciendo posible por ello su formalización futura en subsiguientes fases de construcción teórica.

En su estadio actual, el modelo no define el contenido concreto de sus componentes -antecedentes situacionales, representaciones, estados mentales consecuentes- que adopta como postulados básicos (las premisas en una fase pre-axiomática de construcción teórica) para fijar el objeto de estudio. De hecho, tales contenidos son postulados básicos periféricos porque su cambio o modificación no afectaría al núcleo del modelo (Bunge, 2000). En particular, aunque el modelo reconoce y da por supuesto la existencia de contenidos, dimensiones o factores de autoestima, en principio no se compromete con ninguno de ellos, que tendrían aquí tan sólo un carácter metodológico de primitivas o fundamentos a la base de su cuerpo teórico. Por otro lado, las referencias citadas en el presente estudio se han tomado única y exclusivamente como casos ilustrativos que se han tenido en cuenta en la formación del modelo, muy diversos y a veces con cierta distancia conceptual de conformidad con nuestra intención teórica unificadora. Además, dada la novedad (criterio gnoseológico) de nuestro planteamiento, salvo error, no existen o no hemos hallado antecedentes directos a su estructura. 
Finalmente, decimos que el modelo es "sistémico" en un doble sentido. Con este término nos referirnos tanto a su estructura conceptual sistemática con validez interna (Bunge, 2000) cuanto a su funcionamiento como sistema, en concreto un sistema con retroalimentación. Téngase presente que este tipo de sistemas cubre diversas funciones de autorregulación en el hombre (von Bertalanffy, 1989).

\section{Modelo Propuesto}

Se define la autoestima como un sistema abierto a las interacciones de la persona con su medio y autorregulado, cuya actualización y mantenimiento tienen como finalidad optimizar el estado mental del interesado. Si el sistema se desestabilizara, caso de baja autoestima, intentará entonces restaurarse modificando sus elementos componentes. Una disfunción en este proceso por una razón u otra ocasionará malestar subjetivo y probable psicopatología.

El modelo se atiene a los fundamentos teóricos básicos (Bunge, 2000), siendo su carácter esencialmente cognitivo ya que su estructura conceptual comprende tanto representaciones mentales (Pylyshyn, 1980) como procesos cognitivos (Bourne et al., 1979; Broadbent, 1984; Fodor, 1983; Lachman et al., 1979; Schank, 1984), aunque también incluye conductas de respuesta al ambiente. Su funcionamiento es coherente con las teorías sobre conservación de recursos (Hobfoll, 1989, 2001): autoestima como recurso; de memoria de trabajo (Cowan, 2000): la rumiación autocrítica en la baja autoestima (Kolubinski et al., 2017) que malgasta recursos; de autoeficacia (Bandura, 1986): la incompetencia baja la autoestima; y de aceptación y rechazo interpersonal (Rohner y Carrasco, 2014): la aprobación social refuerza la autoestima; y resulta compatible con el modelo de función ejecutiva autorreguladora (Wells, 2000; Wells y Matthews, 1994, 1996): autoestima como pieza esencial en la autorregulación personal. Su articulación con estos otros constructos en sentido ascendente y descendente refuerza su validez, de conformidad con Bunge (1974). Por lo demás, sus componentes se vinculan con el objeto material de la actual psicología de la mente: esquemas del yo -identidad personal (Metzinger, 2009); estados mentales -autoconsciencia (Noë, 2009); proyecto personal -intencionalidad (Dennett, 1989); y circuito de retroalimentación -causación mental (Varela et al., 2016).

\section{Concepto de Autoestima}

La autoestima se define como el resultado de la previa valoración que hace la persona sobre sí misma, lo cual implica lo siguiente:

a) Al tratarse de una valoración, es necesaria la preexistencia de dos términos previos que la posibilitan y pasan a compararse entre sí para estimar sus diferencias o semejanzas, a saber: (i) el propio yo, o autoconcepto, tal y como es percibido por la persona en cierto momento, y que denominaremos "yo percibido"; y (ii) otro yo que la persona toma como referente o arquetipo de su yo percibido, y que denominaremos “yo modelo". Realmente, en este proceso de valoración se coteja el yo percibido con respecto al yo modelo, siendo ambos representaciones o esquemas mentales. Luego, la autoestima es igual al valor, mérito o demérito, que se otorga alguien a sí mismo tras haberse comparado ambos esquemas. A mayor semejanza entre ellos, mayor autoestima, y viceversa.

b) Al ser una autovaloración, el interesado se entremezcla e involucra a sí mismo en ella, atribuyéndose responsabilidad por su resultado.

Aunque la autoestima corresponda al individuo, sin embargo, su origen y mantenimiento son de naturaleza psicosocial. Se transmite de forma intergeneracional (Steiger et al., 2015), configurándose su desarrollo durante la infancia y adolescencia desde múltiples características del ambiente familiar (Krauss et al., 2019). Johnson (2010) defiende su evolución en dos fases, una autoestima básica, de carácter más afectivo, constituida en primer lugar; y otra autoestima posterior que uno mismo debe ganarse por su actuación. Respecto a las influencias procedentes del ambiente familiar, se ha constatado una relación entre ciertas actuaciones de los 
padres y el desarrollo psicosocial de los hijos (Fuentes et al., 2015; Gallarin et al., 2021). También, la dimensión familiar del yo podría explicarse desde el impacto parental (Martinez et al., 2021).

Siendo fruto de la interacción persona-medio, la autoestima se encaja entre antecedentes y consecuentes, resultando de causas determinadas y produciendo efectos específicos. Su plena inmersión en el seno de una amplia constelación de factores la descubre como parte integrante de un sistema dinámico mayor y más complejo, aludiendo a su forzosa plasticidad. En resumen, sus cualidades esenciales son: (a) forma parte del sistema de interacción persona-medio; (b) se actualiza continuamente al ser contingente con este última, (c) es consecuencia de un previo proceso de comparación, (d) genera efectos particulares, conectados a un proceso de retroalimentación, y (e) su estructura, esto es sus componentes y procesos, constituye un sistema en sí mismo.

Por su parte, el yo modelo exhibe igualmente un perfil propio durante la autovaloración: (a) carácter imperativo: modelo ineludible; (b) rigidez: modelo no modelable; (c) contingencia: los beneficios potenciales del modelo no están garantizados, e (d) inmediatez: activa automáticamente el ciclo de procesamiento.

\section{Yo Modelo como Proyecto}

Sin impedimento de influencias ambientales, cabe que una persona adopte deliberadamente un yo modelo como proyecto personal, a modo de compromiso consigo mismo para ser y comportarse de una determinada manera, opción que equipara la mente humana a un mecanismo propositivo, asunto que atañe a la intencionalidad de nuestros actos, cuestión perteneciente ya al ámbito de la filosofía de la mente (Dennett, 1989; Metzinger, 2009) o filosofía general: el proyecto vital de Ortega y Gasset magníficamente resumido por Marías (1941). Siendo así, entre diversas alternativas asequibles de yo modelo, la persona optaría por una de entre todas ellas o se la confeccionaría a medida. Un reciente estudio (Schick et al., 2020) ha puesto de manifiesto que en la autoestima subyace un factor intrapersonal de origen intrínseco.

Este yo modelo como proyecto estaría ligado igualmente al cumplimiento de ciertas metas personales jalonadas a lo largo de la vida -estudiar, trabajar, consumir-, revelando una dimensión temporal en la autoestima. Por consecuencia, este tipo de autoestima afloraría al compararse la trayectoria de logros obtenidos por el sujeto con respecto a una trayectoria modelo de metas a conseguir previamente ideada. En esta versión longitudinal tendríamos, pues, autoestima como proximidad entre dos trayectorias, frente a autoestima como coincidencia entre dos perfiles de yo en la versión transversal.

\section{Consideraciones sobre el Self}

El modelo, como hemos anticipado en la Introducción, no asume ningún contenido o dimensionalidad en concreto del yo -nuestros conceptos de yo-modelo y yo-percibido, definidos más abajo, se utilizan exclusivamente como términos de comparación. No obstante, admitimos, resultará conveniente exponer aunque sea ello de forma muy breve algunas consideraciones sobre el yo (self), un constructo complejo y de larga tradición en la literatura. Obviamente, las limitaciones de espacio nos impiden una revisión exhaustiva de este concepto, según haya sido entendido por los diversos enfoques teóricos y, menos aún, de los resultados empíricos existentes sobre su dimensionalidad, funcionalidad diferencial de los factores en diversos contextos, e incluso sobre el carácter transcultural del yo, y otras cuestiones afines.

Por un lado, autoconcepto y autoestima son conceptos tan íntimamente relacionados que resultan casi indistinguibles, e incluso se usan de forma indistinta (Pajares y Shuck, 2001; Shavelson y Bolus, 1982). Por otro lado, también existe un amplio debate sobre la dimensionalidad y estructura jerárquica del yo -véase (Baumeister et al., 2003; Chen et al., 2020; Marsh y O’Mara, 2008; Marsh y Shavelson, 1983; Rosenberg 1979) para obtener una amplia visión sobre el asunto. A este último respecto, la estructura teórica de cinco factores -académica, social, emocional, familiar y física- ha venido recibiendo respaldo empírico, incluso en análisis factoriales confirmatorios en diversos países aun con culturas muy distintas (Chen et al., 2020; García et al., 2013; García et al., 2018; Murgui et al., 2012; Tomas y Oliver, 2004). De forma obvia, un enfoque multidi- 
mensional del yo permite predicciones más precisas y una mejor explicación de ciertos trastornos mentales y problemas de conducta específicos (Chen et al., 2020; Fuentes et al., 2020; Gallarin et al., 2021; Garcia et al., 2018; Maiz y Balluerka, 2018).

\section{Estructura}

El modelo consta de dos clases de elementos: (a) componentes: antecedentes situacionales, representaciones y estados mentales consecuentes, y (b) procesos cognitivos: procesan la información entre componentes (ver Gráficos 1 y 2). Todos ellos son momentos necesarios de cada ciclo de autoestimación.

\section{Componentes}

Figura 1. Componentes del modelo de autoestima

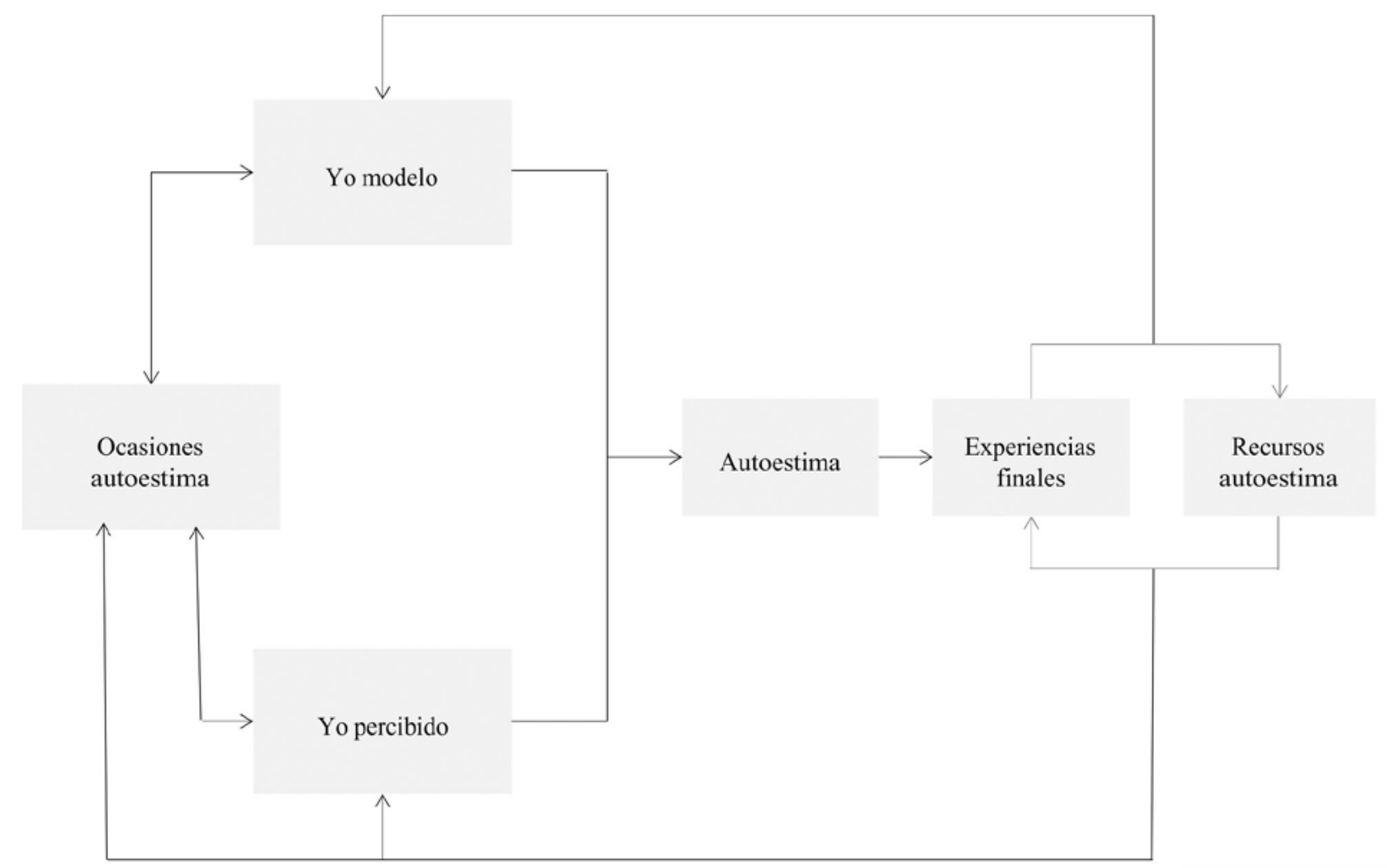

\section{Antecedentes Situacionales}

Ocasiones de autoestima. Aquellas situaciones antecedentes, o aspectos concretos suyos o efectos anticipados, en donde se hallase la persona, y que originen una valoración del yo percibido respecto al yo modelo. Cubren un amplio espectro: situaciones sociales o ejecución de tarea actuales, recuerdo de situaciones pasadas, anticipación de futuras situaciones... Caso de elevar demandas, las ocasiones de autoestima incluyen ya cómo haya respondido el sujeto a las mismas -Johnson (2010) ratifica una autoestima basada en la ejecución. 


\section{Representaciones}

Cuando definimos a continuación los conceptos de yo modelo y yo percibido nos referimos única y exclusivamente a ellos como momentos o términos que intervienen en el proceso de una comparación entre dos representaciones mentales. Como hemos comentado en la Introducción, sus contenidos concretos se suponen pero no se definen, son los postulados básicos en este trabajo.

Yo modelo. Esquema mental que funciona a modo de arquetipo al que equiparar el yo percibido, y que se toma como baremo para valorar a este último. Por definición, será implícito al interesado (Franck et al., 2008), pero quizás pudiera ser explícito si fuera el caso de un proyecto personal o explicitarse durante un proceso de terapia. Su magnetismo descansa en una creencia subyacente en el interesado según la cual asemejarse a este esquema le reportará resultados provechosos; yo modelo no como fin en sí mismo, sino como instrumento: "si soy x, entonces conseguiré y". En consecuencia, el yo modelo se "cargaría" con actitudes, conductas... estimadas adecuadas para obtener ciertos beneficios: "si soy amable, generoso..., entonces conseguiré aceptación social". Por consiguiente, de esta representación emergen expectativas sobre cómo deben resolverse las ocasiones de autoestima. Johnson (2010) habla de una autoestima que debe ganarse uno por sus logros (earning).

Su contenido se desplegaría como un perfil ideal de cualidades relativas al aspecto personal, competencia social o en ejecución de tareas ..., y éxitos personales -académicos, profesionales, sociales-, autocontrol, o autorrespeto en asuntos morales (Clucas, 2020). Hay resultados que así lo atestiguan: la autoestima depende de aquellos principios en los que se base (Pyszczynski et al., 2004); se engrana con motivaciones básicas que impulsan al individuo al logro de objetivos y competencias (Maxwell y Bachkirova, 2010); juega un papel mediacional en la autoeficacia académica (Bandura, 1986; Batool et al., 2017); la competencia personal es su mejor predictor (Soral y Kofta, 2020); y se vincula con el deseo de estatus (Anderson et al., 2015), cuya frustración cursa con baja autoestima (von Soest et al., 2018). Los rasgos de su perfil estarán jerarquizados, subordinándose unos a otros según su significación en términos absolutos o relativos a situaciones presentes.

Aunque se presume un único yo modelo en la mente de la persona, no deben descartarse dos posibilidades: (i) que el interesado pueda servirse de diferentes yo modelos alternativos, utilizándolos a conveniencia; y (ii) que por influencia social el yo modelo vigente quede neutralizado o incluso suplantado por otro yo modelo ajeno. Pero aun siendo así, ello no invalidaría el funcionamiento del modelo por cuanto que en cada autovaloración puntual intervendría exclusivamente un yo modelo y sólo uno.

Yo percibido. Esquema mental de cómo percibe el interesado su propio yo en un momento o periodo determinados, constituyendo su autoconcepto. En el cuestionario al respecto de Robson (1989) se recogen situaciones o características personales conducentes a una sucesiva autoestimación tal y como tener éxito, autocontrol, personalidad agradable, (no) parecer horrible...

En conexión con la versión longitudinal de la autoestima, este esquema incorporaría "extensiones del yo", expresión referida a los resultados tangibles e intangibles que haya obtenido el sujeto en diversos ámbitos, y cuyo éxito o fracaso se atribuya a sí mismo. Se sabe cómo los factores materialistas influyen en la autoestima (Gupta y Singh, 2019).

Recursos autoestima. Representaciones almacenadas en la memoria del interesado con información de cualquier tipo: social, económica ...- y de la que pudiera valerse instrumentalmente aquél para corregir la discrepancia sufrida entre su yo modelo y yo percibido. La riqueza de estos recursos dependerá obviamente de la formación que haya recibido la persona, los conocimientos adquiridos y la experiencia acumulada.

\section{Estados Mentales Consecuentes}

Autoestima. Estado mental que recoge la consideración y aprecio que se otorga uno a sí mismo tras haberse comparado sus dos esquemas de yo, variando en tonalidad conforme al grado de coincidencia entre ambos: a mayor coincidencia, autoestima o tono más elevado. Las discrepancias con un ideal cursan con baja autoestima 
(Renaud y McConnell, 2007); y los ítems para evaluarla desvelan una comparación tácita: "soy capaz de hacer las cosas tan bien cómo la mayoría de las otras personas" (Rosenberg, 1965, p. 306).

Ya que la autoestima se subordina a la interacción persona-medio, conviene introducir aquí dos conceptos complementarios: (a) autoestima posible: autoestima máxima factible dadas las condiciones actuales de la persona y su medio, sería la autoestima relativa; y (b) autoestima plena: autoestima alcanzada cuando ocurra una perfecta semejanza entre yo percibido y yo modelo, sería la autoestima absoluta. Esta última eventualidad, constituirá un hecho singular en la vida del sujeto y se almacenará con mayor prominencia en su memoria episódica. Acompasándose con la interacción citada, la autoestima se irá actualizando, máxime cuando acuse menoscabo significativo, y a efectos de restaurar todo el sistema: hay efectos a corto plazo del estrés sobre la autoestima (Palmier-Claus et al., 2011); ella misma adolece de fragilidad (Borton et al., 2012); y la incertidumbre sobre la propia autoestima se vincula con la depresión (Luxton y Wenzlaff, 2005).

Experiencias finales. Estados de conciencia que albergarán las consecuencias causadas por la autoestima, estando impregnados del tono emocional correspondiente a cómo haya sido esta última. Decimos "finales" porque cierran un ciclo completo de actualización de la autoestima. Irán oscilando de lo positivo a lo negativo en armonía con los cambios en la autoestima actual, y tendrán efecto retroactivo sobre los esquemas de yo y ocasiones de autoestima.

\section{Observación}

Para incluir la versión longitudinal de la autoestima, los componentes del modelo deben completarse con estos tres nuevos conceptos: (a) Yo modelo de metas: esquema con aquellas metas a conseguir en el tiempo, que el interesado se hubiese marcado para estimarse a sí mismo, (b) Yo percibido de logros: esquema con la trayectoria de sus éxitos y fracasos; y (c) Autoestima por logros: la que se obtenga tras haberse cotejado los esquemas previos.

\section{Procesos}

Un conjunto de procesos cognitivos en serie conecta entre sí los componentes del modelo. Requieren la asistencia de recursos cognitivos y metacognitivos para el tratamiento de la información, y se desencadenan automáticamente una vez dados los antecedentes necesarios. Cumplen los criterios de proceso cognitivo (Rowlands, 2010), y por su categoría son procesos subpersonales -información de salida sólo disponible para el proceso subsiguiente-, excepto cuando anteceden a los estados mentales consecuentes.

Hay tres procesos nucleares que originan a su vez procesos derivados, según detalle: (a) deducción y/o recuperación de información desde el yo modelo: genera expectativas sobre cómo deben resolverse las situaciones antecedentes, y conduce a los subprocesos de identificación de situaciones antecedentes, selección e implementación de conductas e interpretación de las ocasiones de autoestima; (b) atribución interna: asigna responsabilidad personal sobre el resultado de la situación, e inicia el subproceso de valoración del yo percibido; y (c) comparación yo percibido-yo modelo: coteja ambos esquemas asignando la autoestima acorde, y lleva a los subprocesos de activación de las experiencias finales desde la autoestima, y retroalimentación desde las experiencias finales.

\section{Deducción y/o recuperación}

Desde un yo modelo suficientemente rico y multiforme se deducen, o se recuperan directamente de su contenido, estos tres bloques de información: (a) clase de situaciones, o patrón de características suyas, potencialmente pertinentes para la autoestima, (b) repertorio de conductas convenientes a desplegar o inhibir en estas situaciones, y (c) conjunto de reglas de interpretación para juzgar si la situación se ha resuelto o no favorablemente a este esquema. 
Figura 2. Procesos del modelo de autoestima

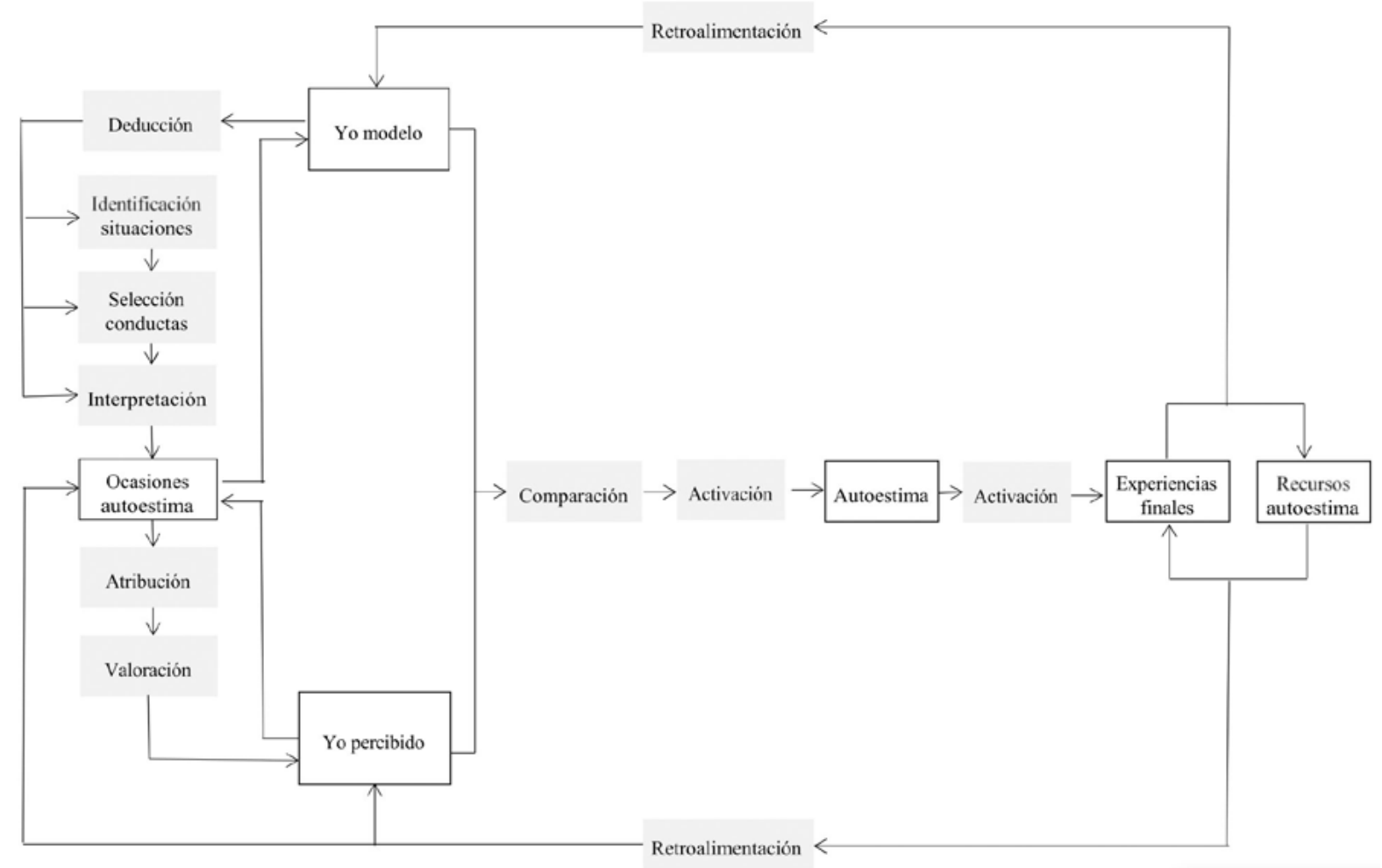

Identificación de situaciones antecedentes. Proceso que toma como entradas: (a) situación antecedente: estímulos ambientales o internos, y (b) patrón de características situacionales pertinentes para la autoestima según el yo modelo, considerándose si hay o no ajuste. Su salida consistirá en aceptar o descartar la situación como ocasión de autoestima. Algunas situaciones elevarán demandas de actuación al sujeto, siendo su cumplimiento requisito para una valoración positiva del yo percibido -el yo influencia este proceso (Schäfer y Frings, 2019) y algunos trastornos lo dificultan (Cella et al., 2018).

Selección e implementación de conducta. Proceso cuyas entradas son: (a) demandas de actuación que pudiera elevar la situación y (b) conductas disponibles para afrontarla convenientemente según el yo modelo; seleccionándose aquella conducta con mayor probabilidad de éxito, que será justo aquélla que simultáneamente atienda las demandas situacionales y las expectativas de éxito del yo modelo. A partir de representaciones y tras identificar un elemento de cierta categoría, se generan metas a manera de reglas de actuación (Pylyshyn, 1984). Su salida será la implementación de la conducta seleccionada, suceso que ocurrirá únicamente cuando el interesado hubiera percibido las susodichas demandas. Es un proceso crucial para la autoestima que conecta las demandas situacionales con una respuesta escogida conforme al yo modelo.

Interpretación de ocasiones de autoestima. Proceso con estas entradas: (a) ocasión de autoestima y (b) criterios del yo modelo para juzgar su resultado; determinándose cómo ha sido el mismo. Su salida será la valoración o devaluación del yo percibido en consonancia con el resultado producido. Aunque indirectamente, aquí se conectan por vez primera los dos esquemas de yo.

\section{Atribución interna}

Proceso que asigna la responsabilidad que el interesado se atribuya a sí mismo sobre el resultado de la ocasión de autoestima, estimándose o desestimándose según haya sido éxito o fracaso. Sus entradas son: (a) 
interpretación de la ocasión de autoestima y (b) conducta implementada en la situación; siendo su salida la valoración concomitante del yo percibido. Un estilo atribucional negativo junto a baja autoestima se asocia a síntomas depresivos (Southall y Roberts, 2002). En ciclos sucesivos de autoestima, tendría lugar una retroalimentación entre citadas entradas y salida.

Figura 3. Detalle valoración del yo percibido

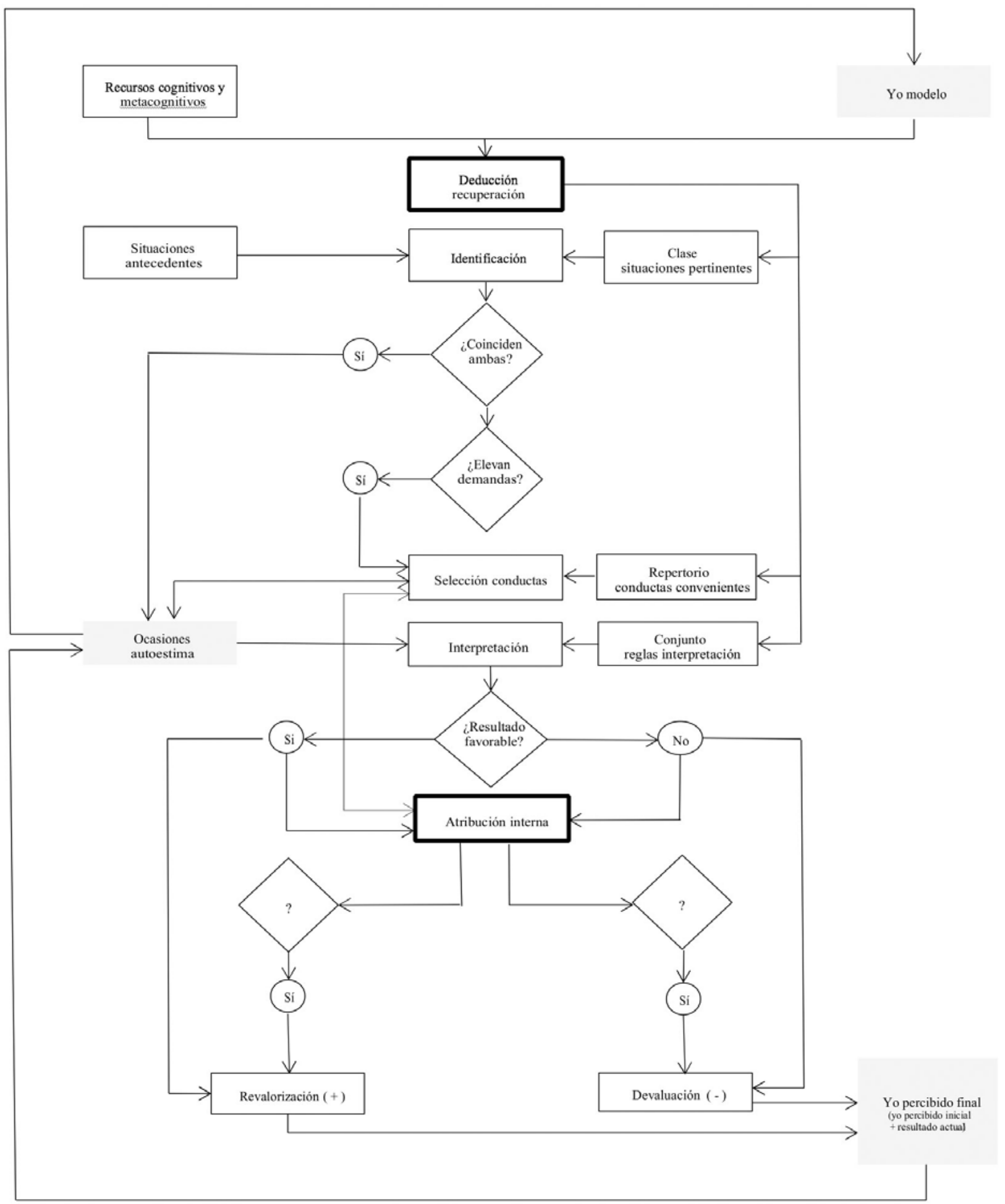




\section{Valoración del yo percibido}

Proceso cuyas entradas son: (a) resultado de la ocasión de autoestima en sí misma, su valoración pasiva, y (b) resultado según la atribución interna efectuada, su valoración activa. Ambas valoraciones se agregarán una a otra en signo y valor. Hablamos de valoración "pasiva" o "activa" refiriéndonos a la participación nula o directa del sujeto en la resolución de la situación. Su salida será un yo percibido final, suma del yo percibido inicial más el valor, de signo negativo o positivo, correspondiente al resultado de la reciente ocasión de autoestima.

\section{Comparación yo modelo-yo percibido}

Proceso donde entran: (a) el yo percibido modelo y (b) el yo percibido final, comparándose entre sí; siendo su salida la activación de un estado mental donde se recoja la autoestima concomitante. Se conectan aquí ya directamente los dos esquemas de yo, evaluándose ambos sobre unos mismos parámetros.

Activación de autoestima. Se sigue inmediatamente del proceso anterior e inicia este estado mental consecuente, siendo accesible a la conciencia del interesado.

Activación de experiencias finales. Proceso cuya entrada y salida son, respectivamente, la autoestima resultante y las consecuencias finales aparejadas. Aquí concluye un ciclo individual de activación y mantenimiento de la autoestima, provocado por cada situación antecedente. Determinados patrones ambientales activan procesos automáticos vinculados a la autoestima (Gawronski y Bodenhausen, 2006).

Retroalimentación. Proceso cuyas entradas son: (a) estado de autoconciencia de las experiencias finales, y (b) recursos de autoestima que disponga y pueda movilizar el sujeto para mejorar su autoestimación; y su salida será el mantenimiento o modificación de las entradas al sistema, retroalimentación positiva y negativa respectivamente.

La secuencia de procesos propuesta guarda coherencia con el paradigma cognitivo y sus aplicaciones terapéuticas para la depresión (Beck et al., 1979) o para el trastorno explosivo intermitente (Gorenstein et al., 2007); basándose, además, en cuestiones de hecho: el antecedente precede a su interpretación, y los resultados son previos a su atribución. Es previsible que la evaluación y seguimiento de estos procesos se probará muy útil en la terapia con pacientes.

\section{Funcionamiento}

Al estar inmersa la autoestima en la dinámica persona-medio, le influyen determinadas situaciones antecedentes, entre otras, y en especial, las relaciones sociales (Pyszczynski et al., 2004); y genera sus particulares consecuencias, siendo, pues, un eslabón intermedio en una cadena causal más extensa. A continuación, examinaremos cómo funciona su actualización y mantenimiento, puntualizando además los componentes del modelo.

\section{Ocasiones de Autoestima}

Representan un conjunto de acontecimientos de una clase dada (Bunge, 1979) e incluyen todos aquellos antecedentes situacionales que desencadenen el proceso de autoestimación. Según se resuelvan, servirá para juzgar si el yo percibido ha satisfecho o no las expectativas del yo modelo (ver Gráfico 3). Se contemplan tres tipos de antecedentes: (a) ocasiones de autoestima externas: cuando el interesado esté percibiendo información ambiental referida a sí mismo o actuaciones suyas: “"recibo muestras de rechazo social”, "he sacado buenas notas"; (b) ocasiones de autoestima interna: estados mentales o sensaciones del interesado, recuerdo 
o anticipación de resultados en situaciones pasadas o futuras: "me encuentro satisfecho con lo que hice", "no podré comportarme adecuadamente"; y (c) ocasiones de autoestima complejas: aquéllas donde se combinen aspectos externos e internos: "me ha felicitado mi jefe, y realmente pienso que lo hice bien". En casos de baja autoestima, los antecedentes más frecuentes conllevan errores, fracaso o rechazo social (Kolubinksi et al., 2016; McManus et al, 2009).

Aunque pudiera parecer que existan ocasiones de autoestima espontáneas, sin intervención alguna del sujeto, el modelo presupone que en todas ellas media una cierta participación suya, aunque la misma hubiera ocurrido hace tiempo -participación remota- o estuviera reducida a mera participación pasiva, de simple presencia física: recibir un gesto de aprobación o rechazo por la calle. La participación del sujeto compromete la subsiguiente atribución de responsabilidad por el resultado de la situación, cobrando por ello especial importancia cómo haya sido su actuación. Esta última afronta una doble dificultad: deberá al mismo tiempo (a) satisfacer las demandas situacionales y (b) resolver la situación conforme a las expectativas del yo modelo. Además, la condición siempre cambiante de la interacción persona-medio elevará continuamente nuevas demandas que obligarán al sujeto a dar distintas respuestas para ir acompasando su actuación con las transformaciones situacionales sobrevenidas, so pena de arriesgar su autoestima. Obviamente, a medida que el yo modelo sea más exigente -obtener no sólo aprobación social, sino inclusive granjearse estatus sociométrico (Anderson et al., 2015)-, será imprescindible movilizar más y más recursos personales, y mayor será también el riesgo de incumplir tales expectativas, ya que unos objetivos personales insatisfechos reducen la autoestima (Lindsay y Scott., 2005).

Dos observaciones finales: en las ocasiones de autoestima: (a) está ya dado e influye un yo percibido inicial sobre el que repercutirá la autovaloración resultante de la ocasión de autoestima en curso, actualizándose aquél a modo de yo percibido final, y (b) por influencia social puede quedar inhibido el vigente yo modelo del interesado, bloqueándose la adecuada selección de conductas y provocando una actuación improcedente en la situación, propiciándose por ello una devaluación del yo percibido final.

\section{Yo Modelo como Principio}

Aunque el yo modelo eleve exigencias, no incluye en sí mismo la manera de cumplirlas. Se postula, sin embargo, que su estructura de contenido funcione a modo de principio para deducir, o quizás recuperar directamente, información substancial para ejecutar eficientemente los procesos cognitivos que operan en la autoestima. En concreto, este esquema facilitaría información para: (a) identificar aquellas situaciones antecedentes que sean ocasiones de autoestima; (b) seleccionar e implementar o inhibir conductas en la ocasión de autoestima actual; e (c) interpretar el resultado logrado en cada situación, según ciertas reglas. La interacción de las diferentes ocasiones de autoestima con el yo modelo pulsará diversas partes del esquema impulsando sendas secuencias de transformación de la información. Procesos cognitivos tales como el priming (Cowan, 2000) facilitan la activación de esquemas desde ciertos estímulos. Su eficacia en cuanto esquema se cifra en proveer: (a) un conjunto de patrones situacionales pertinentes fijados con claridad, (b) un repertorio amplio y versátil de conductas a implementar en las diversas ocasiones que surjan, y (c) unas consistentes reglas de interpretación de los resultados.

Ilustraremos este funcionamiento con un sencillo ejemplo: si "ser persona agradable" figurara en el yo modelo, entonces una situación antecedente de reunión social se identificaría como ocasión de autoestima en compañía de sus demandas, y una actuación que supusiese "conversar animadamente en el grupo" se seleccionaría como opción de conducta adecuada para recibir las simpatías de los asistentes y asegurarse así la autoestima.

\section{Experiencias Finales}

Estas experiencias funcionan como término donde se completa un ciclo aislado de autoestimación -antecedente más secuencia de procesos-, consistiendo en estados de conciencia que recogen las consecuencias últimas de la autoestima. Según sea ésta, se impregnarán de un tono afectivo que oscilará entre un máximo 
positivo, experiencias óptimas, y un máximo negativo, experiencias pésimas; siendo en algunos casos vivencias verdaderamente intensas. Conforme sea su naturaleza e intensidad, llevarán a un proceso retroactivo de mantenimiento o modificación de los componentes del sistema.

En lo positivo, son experiencias de autosatisfacción, autoconfianza, autoaceptación y autocuidado (Jacob et al., 2010), e irán de un bienestar psicológico con sentimientos de alegría o satisfacción a sentimientos de orgullo y autocomplacencia indicativos de una mayor realización o plenitud personal: la autoestima predice sentimientos de estatus e inclusión (Benson y Giacomin, 2020). En su aspecto fenoménico, se manifestarán al interesado como una sensación de despreocupación por haber alcanzado una especie de meta -el yo percibido se volvería "transparente", en el sentido positivo otorgado a este término por Metzinger (2009)- o de coherencia interna entre sus dimensiones cognitiva, emocional y conductual (Duro, 2005). Liberarán recursos cognitivos o facilitarán su acceso para otras tareas o proyectos, ya que aquellas personas con creencias positivas sobre sí mismas se implican más en procesos de resolución de problemas (Roberts et al., 2020); amortiguando las cargas mentales de la ansiedad una autoestima elevada (Pyszczynski et al., 2004).

En su polo negativo, se experimentará autodesconfianza, autorechazo, autocrítica ..., estados coloreados por sentimientos acordes -tristeza, abatimiento, remordimientos-, que producen incapacidad. La recurrente rumiación autocrítica en la baja autoestima (Kolubinski et al., 2017, 2019; Sowislo y Orth,2013) malgasta la capacidad limitada de la memoria de trabajo (Cowan, 2000) en un intento estéril de restaurar aquélla -la tristeza causa episodios de cuestionamiento rumiador (Roberts et al., 2020). La baja autoestima inhibe igualmente la capacidad de percepción y acción del sujeto, teniendo presente que la autoestima media el afrontamiento de situaciones (Kurtovic et al., 2018) -una baja autoestima dificulta la percepción de las señales corporales (Cella et al., 2019), y se sigue de complicaciones, tales como el resentimiento que correlaciona negativamente con ella (Murillo y Salazar, 2019) y de trastornos mentales. Constituye un factor de vulnerabilidad para la depresión (Franck et al., 2008; Steinberg et al., 2007), también en lo relativo a los tipos de autoestima (Johnson, 2010), exacerbando los defectos y fracasos personales (Fennell, 2004), y llegando incluso a producir aquel trastorno (Beck et al., 1979). Paralelamente, una preocupación excesiva por la aceptación social, muy frecuente en la baja autoestima, conlleva procesos de vigilancia asociados a la ansiedad (Beck et al., 1985), sin olvidar que la variabilidad de la autoestima, junto a otros factores, genera síntomas paranoides (Palmier-Claus et al., 2011).

\section{Retroalimentación}

Un circuito de retroalimentación será activado por las experiencias finales para mantener o modificar las entradas, ya en subsiguientes ciclos de autoestima. Funcionará a corto plazo ante cada ocasión de autoestima actual, y a medio y largo plazo para un mantenimiento más duradero. Cuando la discrepancia entre los esquemas de yo hubiese superado cierto umbral, la experiencia negativa concomitante actuará como acicate incitando al interesado a reducir aquélla, dado que la disonancia cognoscitiva potencia siempre una motivación correctora (Festinger, 1957); aunque a veces esta corrección se desvirtúe: serían las reglas de vida patológicas (Fennell, 2004). Su efecto retroactivo operará en dos ámbitos: (a) bucle interno: circuito que interconecta las experiencias finales con el yo modelo y el yo percibido para efectuar cambios en estos esquemas, a modo de mecanismo de autorregulación; y (b) bucle externo: circuito que interconecta las experiencias finales con la percepción y actuación en las ocasiones de autoestima con vistas a optimizar estas últimas, a manera de estrategia adaptativa. Obviamente, la eficacia de la retroalimentación dependerá del monto de recursos de autoestima que pueda movilizar el interesado.

Ocasiones de autoestima que encierren una influencia social intencionada -consejo, rechazo- o casual -exposición fortuita a modelos de yo más atractivos- actuarán asimismo mediante el bucle interno cambiando los esquemas preexistentes de manera temporal o permanente. Debe distinguirse retroalimentación de influencia: allí se corrige una discrepancia y aquí se modifican directamente esquemas preexistentes: alguien impone un yo modelo, una situación anula el yo modelo vigente. Recuérdese que ambos esquemas de yo muestran permeabilidad a los influjos del medio interno -estados de ánimo, sensaciones propioceptivas- y del medio ambiente externo -ocasiones de autoestima. 


\section{Recursos de Autoestima}

Para apaciguar el malestar de las experiencias finales negativas, el interesado necesitará valerse de todos aquellos recursos de autoestima que tenga a su alcance: cognitivos, metacognitivos, sociales, económicos... Una reinterpretación de las situaciones antecedentes o reatribución de su resultado son ejemplos de recursos cognitivos. No puede descartarse que el acceso a estos recursos pueda estar bloqueado por la propia desestimación, toma de decisiones inhibida por falta de confianza en uno mismo.

En especial, serán apropiadas aquí por diferentes razones: (a) habilidades sociales tales como mostrar empatía o prestar apoyo social: aquellos con baja autoestima tienden a congraciarse más con terceros (Schmitte et al., 2019); (b) capacidades de autorregulación para ajustar el sistema de autoestima, en línea con el modelo S-REF (Wells y Matthews, 1994); (c) recursos metacognitivos -reorientar la atención, vigilar las situaciones antecedentes (Wells, 2009); e, incluso, para algunos perfiles de yo modelo (d) recursos económicos o de influencia social para alcanzar ciertos puestos profesionales, contratar servicios o adquirir bienes patrimoniales: las intervenciones estéticas mejoran la autoestima (Richard et al., 2018).

\section{Aplicaciones Clínicas}

El modelo con su carácter analítico posibilita explicar aspectos destacados de los trastornos mentales y el funcionamiento de las terapias existentes para la baja autoestima. Desde sus principios de funcionamiento, se postula que esta última emerge de dos diferenciales básicos: (a) entre el yo modelo y el yo percibido, y (b) entre los recursos disponibles y necesarios para recuperar la autoestima. En cualquier caso, el paciente quedaría sumido en un conflicto medios-fines, en cuyo origen y mantenimiento tendrán parte factores externos -ambiente familiar, influencia social- e internos -ambición personal, desviación social.

\section{Psicopatología}

La baja autoestima acompaña a otros trastornos mentales: alimenticios (Chang 2020), de ansiedad, problemas de conducta y personalidad (Jacob et al., 2010; Pérez-Gramaje et al., 2019), ciertas psicosis (Hall y Tarrier, 2003); y, como factor de vulnerabilidad personal (Butler et al., 1994; Franck et al., 2008; Sowislo y Orth, 2013; Steiger et al., 2015) puede suministrar una base sobre la que se cimente la estructura patológica de otros cuadros: una autoestima consistente predice el curso de la terapia para la depresión (Eberl et al., 2018), y su variabilidad induce mejoras en tratamientos de personalidad (Cummings et al., 2012).

El modelo cifra su etiología en uno o varios de estos defectos: (a) vicios en la configuración de los esquemas del yo, (b) procesos cognitivos de autoestima sesgados o inhibidos por una u otra causa: una autoestima variable llevaría a externalizar el resultado de los acontecimientos (Palmier-Claus et al., 2011), y (c) falta de una retroalimentación negativa eficaz.

Ilustraremos lo anterior con algunos ejemplos, separándolos por componentes y procesos: (a) componentes: un yo percibido negativo quedaría como cristalizado en la mente de la persona debido a situaciones de abuso, acoso, negligencia familiar... (McManus et al., 2009) o estigmatización, cuya internalización daña la autoestima (Jahn et al., 2020), bloqueándose su oportuna actualización por actuaciones exitosas, un cambio terapéutico indispensable (Ellis, 1996); un sobreestimación del yo percibido resultaría igualmente perjudicial: el narcisismo vulnerable correlaciona negativamente con la autoestima (Rohmann et al., 2019); (b) procesos: sesgos al identificar situaciones antecedentes, las personas con baja autoestima tienden a comparaciones sociales ascendentes (Parker et al., 2013); o malinterpretación de expectativas, transformando aspiraciones legítimas en necesidades perturbadora (Ellis y Grieger, 1986); conflicto entre las finalidades de autorregulación y adaptación, a cargo, respectivamente, de los bucles de interno y externo de retroalimentación: la autoestima media el conflicto entre familia y trabajo (Innstrand et al., 2010). 


\section{Psicoterapia}

Como adelantamos anteriormente, existen numerosas terapias al uso para la baja autoestima, algunas de reciente implantación como la derivada del enfoque metacognitivo (Kolubinski et al., 2016, 2018, 2019), a saber: terapia cognitivo-conductual individual con adultos (Cummings et al., 2012; Hall y Tarrier, 2003; McManus et al., 2009; Pack y Condren, 2014; Parker et al., 2013; Waite et al., 2012; Whelan et al., , 2007), adolescentes (Taylor y Montgomery, 2007) y niños (Wanders et al., 2008), también en grupo (Beattie y Beattie, 2018) y en coexistencia con otros trastornos (Jacob et al., 2010; (Pack y Condren, 2014; Whelan et al., 2007); terapia conductual dialéctica (Roepke et al., 2011); terapia racional-emotiva, inclusive integrada con otras terapias (Roghanchi et al., 2013); técnica EMDR (Wanders et al., 2008); enfoque mindfulness (Fennell, 2004); técnicas de coaching (Maxwell y Bachkirova, 2010); o inclusive la terapia del rol fijo (Kelly, 1955), originalmente como tratamiento para la personalidad; existiendo, asimismo, estudios comparativos entre diversas terapias (Wanders et al., 2008).

El modelo puede contribuir a perfeccionar la aplicación de estas terapias, y sienta las bases para un proyecto terapéutico propio a partir de su estructura y funcionamiento: explicitando el yo modelo, mejorando la detección de ocasiones de autoestima. De cualquier manera, se sostiene que la autoestima se recuperará única y exclusivamente optimizando la relación persona-ambiente de cara a incrementar las ocasiones de autoestima que terminen con buena salida.

\section{Conclusiones}

Entre las aportaciones del modelo propuesto, destacaríamos su carácter integrado -completando en un todo conceptos y resultados hasta ahora dispersos- y analítico, que permite explicar la actualización y mantenimiento de la autoestima en tiempo real; así como su potencial para desarrollar futuras aplicaciones clínicas en psicopatología y psicoterapia; su coherencia con otras teorías y constructos psicológicos; y su origen en resultados en este campo de investigación. Dentro de sus limitaciones, entendemos que todavía falta, entre otras cosas, una adecuada articulación de las versiones transversal y longitudinal de la autoestima, y el dilucidar cómo se articulan los aspectos automáticos y deliberados del yo modelo.

A nuestro juicio, en futuros trabajos deberá abordarse: (a) la necesaria formalización del modelo y su contrastación empírica; (b) una exposición amplia y ordenada de un programa de tratamiento para evaluar y ajustar adecuadamente los diversos componentes y procesos cognitivos intervinientes, según hemos visto, en la actualización y mantenimiento de la autoestima; así como (b) un posterior análisis comparativo de su eficiencia clínica respecto de otras terapias actualmente existentes.

\section{Referencias}

Anderson, C., Hildreth, J. A. D. y Howland. L. (2015). Is the desire for status a fundamental human motive? A Review of the Empirical Literature. Psychological Bulletin, 141(3), 574-601. https://psycnet.apa.org/ doi/10.1037/a0038781

Bandura, A. (1986). Social foundations of thought and action. Prentice-Hall.

Batool, S. Sh., Khursheed, S. y Govt, H. J. (2017). Academic procrastination as a product of low self-Esteem: A mediational role of academic self-efficacy. Pakistan Journal of Psychological Research, 32(1), 195-211. https://doi.org/10.1111/ajpy.12280

Baumeister, R. F., Campbell, J. D., Krueger, J. I. y Vohs, K. D. (2003). Does high self-esteem cause better performance, interpersonal success, happiness, or healthier lifestyles? Psychological Science in the Public Interest, 4(1), 1-44. https://doi.org/10.1111/1529-1006.01431

Beattie, S. y Beattie, D. (2018). An investigation into the efficacy of a cognitive behavioural therapy group for low self-esteem in a primary care setting. The Cognitive Behaviour Therapist, 11, Artículo e12. https://doi. org/10.1017/S1754470X18000168 
Beck, A. T., Emery, G. y Greenberg, R. L. (1985). Anxiety disorders and phobias. Basic Books.

Beck, A. T., Rush, J., Shaw, B. y Emery, G. (1979). Cognitive therapy of depression. The Guilford Press.

Benson, A. J. y Giacomin, M. (2020). How self-esteem and narcissism differentially relate to high and (un) stable feelings of status and inclusion. Journal of Personality, 88(6), 1177-1195. https://doi.org/10.1111/ jopy. 12565 .

Borton, J. L. S., Crimmins, A. E., Ashby, R. S. y Ruddiman, J. F. (2012). How do individuals with fragile high self-esteem cope with intrusive thoughts following ego threat? Self and Identity, 11(1), 16-35. https://doi.or $\mathrm{g} / 10.1080 / 15298868.2010 .500935$

Bourne, L. E., Dominowski, R. L., Loftus y Loftus, E. F. (1979). Cognitive processes. Prentice Hall.

Bridge, L., Smith, P. y Rimes, K. A. (2019). Sexual orientation differences in the self-esteem of men and women: A systematic review and meta-analysis. Psychology of Sexual Orientation and Gender Diversity, 6(4), 433-446. https://doi.org/10.1037/sgd0000342

Broadbent, D. E. (1984). Mental models. Quarterly Journal of Experimental Psychology, 36A, 673-681.

Bunge, M. (1974). Treatise on Basic Philosophy. Semantics I: Sense and Reference (Vol. 1). Reidel Publishing Company.

Bunge, M. (1979). Treatise on Basic Philosophy. Ontology II: A World of Systems (Vol. 4). Reidel Publishing Company.

Bunge, M. (2000). La investigación científica. Siglo XXI Editores.

Butler, A. C., Hokanson, J. E. y Flynn, H. A. (1994). A comparison of self-esteem lability and low trait self-esteem as vulnerability factors for depression. Journal of Personality and Social Psychology, 66(1), 166-177. https://doi.org/10.1037/0022-3514.66.1.166

Cella, S., Cipriano, A., Giardiello, C. y Cotrufo, P. (2019). Relationships between self-esteem, interoceptive awareness, impulse regulation, and binge eating. Path analysis in bariatric surgery candidates. Clinical Neuropsychiatry, 16(5-6), 213-220. https://doi.org/10.36131/ clinicalnpsych2019050604

Chang, O. (2020). The stakes of self-worth: Examining contingencies of self-worth to clarify the association between global self-esteem and eating disturbances in college women. Journal of Clinical Psychology, 76(12), 6-24. https://doi.org/10.1002/jclp.23006

Chen, F., Garcia, O. F., Fuentes, M. C., Garcia-Ros, R. y Garcia, F. (2020). Self-concept in china: validation of the chinese version of the five-factor self-concept af5 questionnaire. Symmetry, 12(5), Artículo 798. https:// doi.org/10.3390/sym12050798

Clucas, C. (2020). Understanding self-respect and its relationship to self-esteem. Personality and Social Psychology Bulletin, 46(6) 839-855. https://doi.org/10.1177/0146167219879115

Cowan, N. (2000). The magical number-4 in short term memory: A reconsideration of mental storage capacity. Behavioral and Brain Sciences, 24(1), 87-185. https://doi.org/10.1017/S0140525X01003922

Cummings, J. A., Hayes, A. M., Cardaciotto, L. A. y Newman, C. F. (2012). The dynamics of self-esteem in cognitive therapy for avoidant and obsessive-compulsive personality disorders: An adaptive role of self-esteem variability? Cognitive Therapy Research,36, 272-281. https://doi.org/10.1007/s10608-011-9375-X

Dennett, D. C. (1989). The intentional stance. The MIT Press.

Duro, A. (2005). Calidad de Vida Laboral y Psicología Social de la Salud Laboral: hacia un modelo de componentes comunes para explicar el bienestar laboral psicológico y la salud mental laboral de origen psicosocial. Fundamentos teóricos. Revista del Ministerio de Trabajo y Asuntos Sociales. Serie Economía y Sociología, $56,15-56$.

Eberl, C., Winkler, I., Pawelczack, S., TroÈbitz, E., Rinck. M., Becker, E. S. y Lindenmeyer, J. (2018). Self-esteem consistency predicts the course of therapy in depressed patients. PLOS ONE, 3(7), Artículo e0199957. https://doi.org/10.1371/journal.pone.0199957

Ellis, A. (1996). Better, deeper and more enduring brief therapy: the rational emotive behavior therapy approach. Psychology Press.

Ellis, A. y Grieger, R. (1986). Handbook of rational-emotive therapy. Springer Publishing Company.

Fennell, M. (2004). Depression, low self-esteem and mindfulness. Behaviour Research and Therapy, 42(9), 1053-1067. https://doi.org/10.1016/i.brat.2004.03.002 
Festinger, L. (1957). A theory of cognitive dissonance. Stanford University Press.

Fodor, J. A. (1983). The modularity of mind. M. I. T. Press.

Franck, E., De Raedt, R. y De Houwer, J. (2008). Activation of latent self-schemas as a cognitive vulnerability factor for depression: The potential role of implicit self-esteem. Cognition and Emotion, 22(8), 1588-1599. https://doi.org/10.1080/02699930801921271

Fuentes, M. C., Alarcon, A., Garcia, F. y Gracia, E. (2015). Use of alcohol, tobacco, cannabis and other drugs in adolescence: Effects of family and neighborhood. Anales de Psicología, 31(3), 1000-1007. https://doi. org/10.6018/analesps.31.3.183491

Fuentes, M. C., Garcia, O. F. y Garcia, F. (2020). Protective and risk factors for adolescent substance use in Spain: Self-esteem and other indicators of personal well-being and ill-being. Sustainability, 12(15), 5962. https://doi.org/10.3390/su12155962

Gallarin, M., Torres-Gomez, B. y Alonso-Arbiol, I. (2021). Aggressiveness in adopted and non-adopted teens: The role of parenting, attachment security, and gender. International Journal of Environmental Research and Public Health, 18(4), 2034. https://doi.org/10.3390/ijerph18042034

García, F., Gracia, E. y Zeleznova,A. (2013). Validation of the English version of the Five-Factor Self-Concept Questionnaire. Psicothema, 25(4), 549-555. https://doi.org/10.7334/psicothema2013.33

Garcia, F., Martinez, I., Balluerka, N., Cruise, E., Garcia, O. F. y Serra, E. (2018). Validation of the Five-Factor Self-Concept Questionnaire AF5 in Brazil: Testing factor structure and measurement invariance across language (Brazilian and Spanish), gender and age. Frontiers in Psychology, 9, Artículo 2250. https://doi. org/10.3389/fpsyg.2018.02250

Gawronski, B. y Bodenhausen, G. V. (2006). Associative and propositional processes in evaluation: An integrative review of implicit and explicit attitude change. Psychological Bulletin, 132(5), 692-731. https://doi. org/10.1037/0033-2909.132.5.745

Gorenstein, E. E., Tager, F. A., Shapiro, P. A., Monk, C. y Sloan, R. P. (2007). Cognitive-behavior therapy for reduction of persistent anger. Cognitive and Behavioral Practice, 41(2), 168-184. https://doi.org/10.1016/j. cbpra.2006.07.004

Gupta, N. y Singh, R. A. (2019). Role of materialism in influencing self-esteem. Journal of Projective Psychology y Mental Health, 26(1), 51-55.

Hall, P. L. y Tarrier, N. (2003). The cognitive-behavioural treatment of low self-esteem in psychotic patients: a pilot study. Behaviour Research and Therapy, 41(3), 317-332. https://doi.org/10.1016/S00057967(02)00013-X

Harris, M. A. y Orth, U. (2020). The link between self-esteem and social relationships: A meta-analysis of longitudinal studies. Journal of Personality and Social Psychology: Personality Processes and Individual Differences, 119(6), 1459-1477. https://doi.org/10.1037/pspp0000265

Innstrand, S. T., Langballe, E. M., Espnes, G. A., Aasland, O. G. y Falkum, E. (2010). Personal vulnerability and work-home interaction: The effect of job performance-based self-esteem on work/home conflict and facilitation. Scandinavian Journal of Psychology, 51(6), 480-487. https://doi.org/10.1111/j.14679450.2010.00816.x

Hobfoll, S. E. (1989). Conservation of resources: A new attempt at conceptualizing stress. American Psychologist, 44(3), 513-524. https://doi.org/10.1037/0003-066X.44.3.513

Hobfoll, S. E. (2001). The influence of culture, community, and the nested self in the stress process: Advancing conservation of resources. Applied Psychology, 50(3), 337-421. https://doi.org/10.1111/1464-0597.00062

Huang, Ch. (2010). Mean-level change in self-esteem from childhood through adulthood: Meta-analysis of longitudinal studies. Review of General Psychology, 14(3), 251-260. https://doi.org/10.1037/a0020543

Jacob, G. A., Gabriel, S., Roepke, S., Stoffers, J. M., Lieb, K. y Lammers, C-H. (2010). Group therapy module to enhance self-esteem in patients with borderline personality disorder: A pilot study. International. Journal of Group Psychotherapy, 60(3), 373-387. https://doi.org/10.1521/ijgp.2010.60.3.373

Jahn, D. R., Leith, J., Muralidharan, A., Drapalski, A. L., Hack, S. y Lucksted, A. (2020). The influence of experiences of stigma on recovery: Mediating roles of internalized stigma, self-esteem, and self-efficacy. Psychiatric Rehabilitation Journal, 43(2), 97-105. https://doi.org/10.1037/prj0000377 
Johnson, M. (2010). Depressive styles, self-esteem structure, and health: A dynamic approach to differential vulnerability in self-criticism and dependency. Individual Differences Research, 8(1), 45-66.

Kästner, D., Löwe, B. y Gumz, A. (2019). The role of self--esteem in the treatment of patients with anorexia nervosa-A systematic review and meta-analysis. International Journal of Eating Disorders, 52(2), 101116. https://doi.org/10.1002/eat.22975

Kelly, G. A. (1955). The psychology of personal constructs. Norton.

Kolubinski, D. C., Frings, D., Nikčevićb, A. V., Lawrencea, J. A. y Spada, M. M. (2018). A systematic review and meta-analysis of CBT interventions based on the Fennell model of low self-esteem. Psychiatry Research, 267, 296-305. https://doi.org/10.1016/j.psychres.2018.06.025

Kolubinski, D. C., Marino, C., Nikčevićb, A. V. y Spada, M. M, (2019). A metacognitive model of self-esteem. Journal of Affective Disorders, 256,42-53. https://doi.org/10.1016/j.jad.2019.05.050

Kolubinski, D. C., Nikčevićb, A. V., Lawrencea, J. A. y Spada, M. M. (2017). The metacognitions about self-critical rumination questionnaire. Journal of Affective Disorders, 220, 129-138. https://doi.org/10.1016/j. jad.2017.06.002

Krauss, S., Orth, U. y Robins, R. W. (2020). Family environment and self-esteem development: A longitudinal study from age 10 to 16. Journal of Personality and Social Psychology, 119(2), 457-478. https://doi. org/10.1037/pspp0000263

Kurtovic, A., Vukovic, I. y Gajic, M. (2018). The effect of locus of control on university students' mental health: Possible mediation through self-esteem and coping. The journal of psychology, 152(6), 341-357. https:// doi.org/10.1080/00223980.2018.1463962

Lachman, P., Lachman, J. L. y Butterfield, E. C. (1979). Cognitive psychology and information processes. An introduction. Lawrence Erlbaum.

Lindsay, J. E. y Scott, W. D. (2005). Dysphoria and self-esteem following an achievement event: Predictive Validity of Goal Orientation and Personality Style Theories of Vulnerability. Cognitive Therapy and Research, 29(6), 769-785. https://doi.org/10.1007/s10608-005-9637-6

Luxton, D. D. y Wenzlaff, R. M. (2005). Self-esteem uncertainty and depression vulnerability. Cognition and Emotion, 19(4), 611-622. https://doi.org/10.1080/02699930441000355

Maiz, E. y Balluerka, N. (2018). Trait anxiety and self-concept among children and adolescents with food neophobia. Food Research International, 105, 1054-1059. https://doi.org/10.1016/j.foodres.2017.12.037

Marías, J. (1941). Historia de la filosofía. Biblioteca Revista de Occidente.

Marsh, H.W.y O'Mara, A. (2008). Reciprocal effects between academic self-concept, self-esteem, achievement, and attainment over seven adolescent years: Unidimensional and multidimensional perspectives of self-concept. Personality and Social Psychology Bulletin,34(4), 542-552.https://doi.org/10.1177/0146167207312313

Marsh, H. W. y Shavelson, R. J. (1985). Self-concept: Its multifaceted, hierarchical structure. Educational Psychologist, 20(3), 107-123. https://doi.org/10.1207/s15326985ep2003 1

Martinez, I., Murgui, S., Garcia, O. F. y Garcia, F. (2021). Parenting and adolescent adjustment: The mediational role of family self-esteem. Journal of Child and Family Studies, 30, 1184-1197. https://doi.org/10.1007/ s10826-021-01937-Z

Maxwell, A. y Bachkirova, T. (2010). Applying psychological theories of self-esteem in coaching practice. International Coaching Psychology Review, 5(1), 16-26.

McManus, F., Waite, P. y Shafran, R. (2009). Cognitive-behavior therapy for low self-esteem: A case example. Cognitive and Behavioral Practice, 16(3), 266-275. https://doi.org/10.1016/j.cbpra.2008.12.007

Metzinger, Th. (2009). The ego tunnel. Basic Books.

Murgui, S., Garcia, C., Garcia, Á. y Garcia, F. (2012). Self-concept in young dancers and non-practitioners: Confirmatory factor analysis of the AF5 scale. Revista de Psicología del Deporte, 21, 263-269.

Murillo, J. y Salazar, M.A. (2019). La autoestima, la extraversión y el resentimiento como variables predictoras del bienestar subjetivo. Universitas Psychologica, 18(1), 1-18. https://doi.org/10.11144/Javeriana. upsy 18-1.aerv

Noë A. (2009). Out of our heads: Why you are not your brain, and other lessons from the biology of consciousness. Hill and Wang. 
Orth, U., Erol, R. Y. y Luciano, E. C., (2018). Development of self-esteem from age 4 to 94 years: A meta-analysis of longitudinal studies. Psychological Bulletin, 144(10), 1045-1080. https://doi.org/10.1037/ bu10000161

Pack, S. y Condren, E. (2014). An evaluation of group cognitive behaviour therapy for low self-esteem in primary care. The Cognitive Behaviour Therapist, 7(7), 1-10. https://oi.org/10.1017/S1754470X14000051

Pajares, F. y Schunk, D. H. (2001). Self-beliefs and school success: Self-efficacy, self-concept, and school achievement. En R. J. Riding y S. G. Rayner (Eds.), Self-perception (pp. 239-265). Ablex.

Palmier-Claus, J. E., Dunn, G., Morrison, A. P. y Lewis, S. W. (2011). The role of metacognitive beliefs in stress sensitization, self-esteem variability, and the generation of paranoia. Cognitive Neuropsychiatry, 16(6), 530-546. https://doi.org/10.1080/13546805.2011.561583

Parker, Th. J., Page, A. C. y Hooke, G. R. (2013). The influence of individual, group, and relative self-esteem on outcome for patients undergoing group cognitive-behavioural therapy treatment. British Journal of Clinical Psychology, 52(4), 450-463. https://doi.org/10.1111/bjc.12029

Pérez-Gramaje, A. F., Garcia, O. F., Reyes, M., Serra, E. y García, F. (2019). Parenting styles and aggressive adolescents: Relationships with self-esteem and personal maladjustment. The European Journal of Psychology Applied to Legal Context, 12(1) 1-10. https://doi.org/10.5093/ejpalc2020a1

Pylyshyn, Z. W. (1980). Cognitive representation and de the process-architecture distinction. The Behavioral and Brain Sciences, 3, 154-169.

Pylyshyn, Z. W. (1984). Computation and cognition: Toward a foundation for cognitive science. The Massachusetts Institute of Technology.

Pyszczynski, T., Greenberg, J., Solomon, Sh., Arndt, J. y Schimel, J. (2004). Converging toward an integrated theory of self-esteem: Reply to Crocker and Nuer (2004), Ryan and Deci (2004), and Leary (2004). Psychological Bulletin, 130(3), 483-488. https://doi.org/10.1037/0033-2909.130.3.483

Renaud, J. M. y McConnell, A. R. (2007). Wanting to be better but thinking you can't: Implicit theories of personality moderate the impact of self-discrepancies on self-esteem. Self and Identity, 6(1), 41-50. https:// doi.org/10.1080/15298860600764597

Richard, A., Harbeck, N., Wuerstlein, R. y Wilhelm, F. H. (2018). Recover your smile: Effects of a beauty care intervention on depressive symptoms, quality of life, and self-esteem in patients with early breast cancer. Psycho-Oncology, 28(2), 401-407. https://doi.org/10.1002/pon.4957

Roberts, J. E., Kyunga, Y., Rosenfelda, E. y Leeb, H. J. (2021). Response styles to sad mood and self-esteem as predictors of autobiographical memory: Distinctions between memory specificity and detail. Personality and Individual Differences, 169, Artículo 110142. https://doi.org/10.1016/j.paid.2020.110142

Robson, P. (1989). Development of a new self-report questionnaire to measure self-esteem. Psychological Medicine, 19(2), 513-518. https://doi.org/10.1017/S003329170001254X

Roepke, S., Schröder-Abé. M., Schütz, A., Jacob, G., Dams, A., Vater, A., Rüter, A., Merkl, A., Heuser, I. y Lammers, C. H. (2011). Dialectic behavioural therapy has an impact on self-concept clarity and facets of self-esteem in women with borderline personality disorder. Clinical Psychology and Psychotherapy, 18(2), 148-158. https://doi.org/10.1002/cpp.684

Roghanchi, M., Mohamad, A. R., Mey, S.Ch., Momeni, K. M. y Golmohamadian, M. (2013). The effect of integrating rational emotive behavior therapy and art therapy on self-esteem and resilience. The Arts in Psychotherapy, 40(2), 179-184. https://doi.org/10.1016/j.aip.2012.12.006

Rohmann, E., Hanke, S. y Bierhoff, W. (2019). Grandiose and vulnerable narcissism in relation to life satisfaction, self-esteem, and self-construal. Journal of Individual Differences, 40(4), 194-203. https://doi. org/10.1027/1614-0001/a000292

Rohner, R. P. y Carrasco, M. A. (2014). Teoría de la Aceptación-Rechazo Interpersonal. Acción Psicológica, 11(2), 9-25. https://doi.org/10.5944/ap.11.2.14172

Rosenberg, M. (1965). Society and adolescent child. Princeton University Press.

Rosenberg, M. O. (1979). Conceiving the self. Basic Books.

Rowlands, M. (2010). The new science of the mind. From extended mind to embodied phenomenology. A Bradford Book, The MIT Press. 
Ryeng, M. S., Kroger, J., Martinussen, M. (2013). Identity status and self-esteem: A meta-analysis. An International Journal of Theory and Research, 13(3), 201-213. https://doi.org/10.1080/15283488.2013.799431

Schäfer, S. y Frings, Ch. (2019). Understanding self-prioritization: the prioritization of self-relevant stimuli and its relation to the individual self-esteem. Journal of Cognitive Psychology, 31(8), 813-824. https://doi. org/10.1080/20445911.2019.1686393

Schank, R. C. (Ed.). (1984). Conceptual information processing. North-Holland.

Schick, M. R., Nalven, T. y Spillane, N. (2020). The factor structure of self-esteem and its association with alcohol use in American Indian (AI) adolescents. American Journal of Orthopsychiatry, 90(6), 712-719. https://doi.org/10.1037/ort0000504

Shavelson, R. J. y Bolus, R. (1982). Self-concept: The interplay of theory and methods. Journal of Educational Psychology, 74(1), 3-17. https://doi.org/10.1037/0022-0663.74.1.3

Schmitte, K., Schreurs, B., Segers, M. y Jawajar, I. M. "Jim" (2019). Within-subject variability in ingratiation as a function of self-esteem and time. Journal of Personnel Psychology, 18(4), 216-226. https://doi. org/10.1027/1866-5888/a000237

Shiina, A., Nakazato, M., Mitsumori, M., Koizumi, H. Shimizu, E., Fujisake, M. y Iyo, M. (2005). An open trial of outpatient group therapy for bulimic disorders: Combination program of cognitive behavioral therapy with assertive training and self-esteem enhancement. Psychiatry and Clinical Neurosciences, 59(6), 690-696. https://doi.org/10.1111/j.1440-1819.2005.01438.x

Soral, W. y Kofta, M. (2020). Differential effects of competence and morality on self-esteem at the individual and the collective level. Social Psychology, 51(3), 183-198. https://doi.org/10.1027/1864-9335/a000410

Southall, S. y Roberts, J. F. (2002). Attributional style and self-esteem in vulnerability to adolescent depressive symptoms following life stress: a 14-week prospective study. Cognitive Therapy and Research, 26(5), 563-579. https://doi.org/10.1023/A:1020457508320

Sowislo, J. F. y Orth, U. (2013). Does low self-esteem predict depression and anxiety? A meta-analysis of longitudinal studies. Psychological Bulletin, 139(1), 213-240. https://oi.org/10.1037/a0028931

Steiger, A. E., Fend, H. A. y Allemand, M. (2015). Testing the vulnerability and scar models of self-esteem and depressive symptoms from adolescence to middle adulthood and across generations. Developmental Psychology, 51(2), 236-247. https://doi.org/10.1037/a0038478

Steinberg, J. A., Karpinski, A. y Alloy, L. B. (2007). The exploration of implicit aspects of self-esteem in vulnerability - Stress models of depression. Self and Identity, 6(2-3), 101-117. https://doi. org/10.1080/15298860601118884

Taylor, T. L. y Montgomery, P. (2007). Can cognitive-behavioral therapy increase self-esteem among depressed adolescents? A systematic review. Children and Youth Services Review, 29(7), 823-839. https://doi. org/10.1016/j.childyouth.2007.01.010

Tomás, J. M. y Oliver, A. (2004). Confirmatory factor analysis of a Spanish multidimensional scale of self-concept. Revista Interamericana de Psicología , 38, 285-293.

Varela, F. J., Thompson, E. y Rosch, E. (2016). The embodied mind: Cognitive science and human experience. The MIT Press.

Von Bertalanffy, L. (1989). Teoría general de los sistemas. México: Fondo de Cultura Económica.

Von Soest, T., Wagner, J., Hansen, Th. y Gerstorf, D. (2018). Self-esteem across the second half of life: The role of socioeconomic status, physical health, social relationships, and personality factors. Journal of Personality and Social Psychology, 114(6), 945-958. https://doi.org/10.1037/pspp0000123

Waite, P., McManus, F. y Shafran, R. (2012). Cognitive behaviour therapy for low self-esteem: A preliminary randomized controlled trial in a primary care setting. Journal of Behavior Therapy and Experimental Psychiatry, 43(4), 1049-1057. https://doi.org/10.1016/j.jbtep.2012.04.006

Wanders, F., Serra, M. y de Jongh, A. (2008). EMDR versus CBT for children with self-esteem and behavioral problems: A randomized controlled trial. Journal of EMDR Practice and Research, 2(3), 180-189. https:// doi.org/10.1891/1933-3196.2.3.180

Wells, A. (2009). Metacognitive therapy for anxiety and depression. The Guilford Press.

Wells, A. (2000). Emotional disorders and metacognition: Innovative cognitive therapy. Wiley. 
Wells, A. y Matthews, G. (1994). Attention and emotion: A clinical perspective. Erlbaum.

Wells, A. y Matthews, G. (1996). Modelling cognition in emotional disorder: The S-REF model. Behaviour Research and Therapy, 32(11-12), 867-870. https://doi.org/10.1016/S0005-7967(96)00050-2

Whelan, A., Haywood, P. y Galloway, S. (2007). Low self-esteem: Group cognitive behaviour therapy. British Journal of Learning Disabilities, 35(2), 125-130. https://doi.org/10.1111/j.1468-3156.2007.00418.X

Zuckerman, M., Li, Ch. y Hall, J. A. (2016). When men and women differ in self-esteem and when they don't: A meta-analysis. Journal of Research in Personality, 64, 34-51. https://doi.org/10.1016/j.jrp.2016.07.007

Artículo recibido: $16 / 09 / 2020$

Artículo aceptado: 22/09/2021 